\title{
CT of hepatocellular carcinoma in non-alcoholic fatty liver disease: imaging characteristics and inter- rater agreement
}

\author{
Ishan Garg ${ }^{1, \#, ~ S c o t t ~ M . ~ T h o m p s o n ~}{ }^{1, \#, ~ S h a n n o n ~ P . ~ S h e e d y ~}{ }^{1}$, Taofic Mounajjed ${ }^{2}$, Ashish Khandelwal', Eric C. \\ Ehman', Candice A. Bookwalter ${ }^{1}$, Sudhakar K. Venkatesh ${ }^{1}$ \\ 'Department of Radiology, Mayo Clinic College of Medicine, Rochester, MN 55905, USA. \\ ${ }^{2}$ Department of Laboratory Medicine and Pathology, Mayo Clinic College of Medicine, Rochester, MN 55905, USA \\ \#Co-equal first authors.
}

Correspondence to: Dr. Sudhakar K. Venkatesh, Department of Radiology, Mayo Clinic College of Medicine, $2001^{\text {st }}$ St SW, Rochester, MN 55905, USA. E-mail: venkatesh.sudhakar@mayo.edu

How to cite this article: Garg I, Thompson SM, Sheedy SP, Mounajjed T, Khandelwal A, Ehman EC, Bookwalter CA, Venkatesh SK. CT of hepatocellular carcinoma in non-alcoholic fatty liver disease: imaging characteristics and inter-rater agreement. Hepatoma Res 2019;5:39. http://dx.doi.org/10.20517/2394-5079.2019.009

Received: 16 Aug 2019 First Decision: 29 Sep 2019 Revised: 7 Oct 2019 Accepted: 10 Oct 2019 Published: 17 Oct 2019

Science Editor: Dalbir Sandhu Copy Editor: Cai-Hong Wang Production Editor: Jing Yu

\begin{abstract}
Aim: To determine the computed tomography (CT) features of non-alcoholic fatty liver disease (NAFLD) associated hepatocellular carcinoma (HCC).

Methods: In this institutional review board approved study, we reviewed 38 patients with NAFLD (68.4\% male; mean age 63 years) with histology confirmed HCC and triphasic liver CT. CT images were independently reviewed by four readers blinded to clinical and pathology data. The reviewers assessed HCC for arterial phase hyper enhancement (APHE), portal venous phase washout (PVWO), delayed phase washout (DPWO), and enhancing capsule. Features of cirrhotic morphology and portal hypertension $(\mathrm{PH})$ were also evaluated. The final $\mathrm{CT}$ features were determined by majority and a fifth reader reviewed cases lacking majority. Inter-rater agreement was determined by prevalenceadjusted kappa.
\end{abstract}

Results: Mean HCC size was $3.6 \pm 2.8 \mathrm{~cm}$ (range, $1.1-16.0 \mathrm{~cm}$ ). The HCCs showed APHE in 92.1\%, PVWO in 55.3\%, DPWO in $81.6 \%$, and enhancing capsule in $44.7 \%$. Cirrhotic morphology was present in $65.8 \%$ and PH in $63.2 \%$. Inter-rater agreement was moderate to almost perfect for APHE (0.74-1.0), cirrhosis (0.79-0.89), and PH (0.790.95), weak to perfect for DPWO (0.47-0.95) and poor for PVWO (0-0.42).

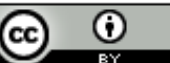

(C) The Author(s) 2019. Open Access This article is licensed under a Creative Commons Attribution 4.0 International License (https://creativecommons.org/licenses/by/4.0/), which permits unrestricted use, sharing, adaptation, distribution and reproduction in any medium or format, for any purpose, even commercially, as long as you give appropriate credit to the original author(s) and the source, provide a link to the Creative Commons license, and indicate if changes were made.

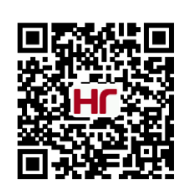


Conclusion: NAFLD associated HCC demonstrate less frequent portal venous washout on CT which may affect their imaging diagnosis.

Keywords: Hepatocellular carcinoma, computed tomography, fatty liver, inter-rater agreement, non-alcoholic fatty liver disease

\section{INTRODUCTION}

Hepatocellular carcinoma (HCC) is the second most common cause of cancer related death worldwide, with increasing mortality rates in Europe, North America, South America and Africa ${ }^{[1,2]}$. Imaging plays a pivotal role in management of HCC and is an established method for diagnosis with radiological hallmarks on contrast enhanced multiphase computed tomography (CT) or magnetic resonance imaging (MRI). The imaging hallmark features include arterial phase hyper enhancement (APHE), portal venous phase washout (PVWO) and/or delayed phase washout (DPWO), and presence of enhancing capsule. Based on some or all of the three features, several guidelines have been developed for the non-invasive imaging diagnosis and standardization in reporting of observations suspicious for HCC such as European Association for the Study of the Liver (EASL), European Organization for Research and Treatment of Cancer, Organ Procurement and Transplantation Network (OPTN), American Association for the Study of Liver Diseases (AASLD) and Liver Imaging Reporting and Data System. It should be noted that EASL does not recognize capsule as a major imaging feature of HCC. However, these guidelines have only been validated in patients with most commonly recognized risk factors for development of HCC including, alcoholic cirrhosis and chronic viral hepatitis and not in non-alcoholic fatty liver disease (NAFLD) $)^{[3-8]}$.

Although most HCCs (75\%-90\%) develop in cirrhosis resulting from chronic hepatitis B or C infections and alcoholic injury ${ }^{[9]}$, an estimated $4 \%-22 \%$ of HCC occur in the setting of NAFLD ${ }^{[10-12]}$. NAFLD has now become the most common cause chronic liver disease in developed countries ${ }^{[13,14]}$. Given its increasing prevalence worldwide, NAFLD may become the most common chronic liver disease associated with HCC.

NAFLD is a spectrum of disease ranging simple non-alcoholic fatty liver (NAFL) to non-alcoholic steatohepatitis (NASH) that can progress to cirrhosis ${ }^{[15]}$. NAFL is considered to have minimal risk of progression to cirrhosis and liver failure, while NASH can progress to cirrhosis, liver failure and develop HCC. NASH is thought to be a common underlying cause of cryptogenic cirrhosis as the patients with cryptogenic cirrhosis are comprised mostly of patients with metabolic risk factors including obesity, metabolic syndrome and diabetes ${ }^{[15]}$, but other etiologies such as burnt-out autoimmune hepatitis and occult alcoholism may also result in cryptogenic cirrhosis ${ }^{[16-18]}$. HCCs are known to occur in patients with NAFLD in the absence of cirrhosis ${ }^{[10,19-21]}$ and these HCCs may not meet the imaging criteria based on the current guidelines including LIRADs ${ }^{[22]}$.

The effect of hepatic steatosis in NAFLD on the imaging features of HCC has not yet been fully explored. For example, hepatic steatosis may decrease the liver attenuation on CT and as a result, washout observation may be absent or less conspicuous which could render the LIRADSv2017 imaging criteria not applicable $e^{[22-25]}$. HCCs can also occur in the absence of cirrhosis in patients with NAFLD as mentioned earlier. Therefore based on these premises, the purpose of our study was to determine the major imaging features of HCC on multiphase CT and the inter-observer agreement in patients with NAFLD.

\section{METHODS}

In this institutional review board (IRB) approved (ID: 15-004925), HIPPA-compliant retrospective study. Written informed consent for retrospective review of data was waived. 


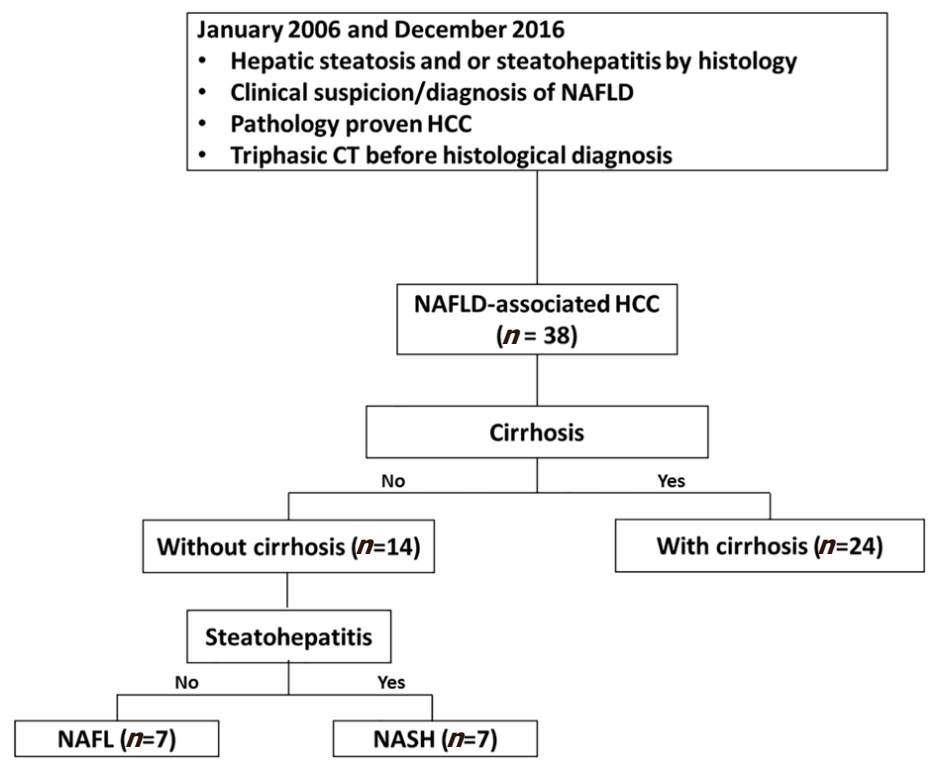

Figure 1. Patient selection flow diagram. CT: computed tomography; HCC: hepatocellular carcinoma; NAFLD: non-alcoholic fatty liver disease; NAFL: non-alcoholic fatty liver; NASH: nonalcoholic steatohepatitis

\section{Patient selection}

We reviewed our institutional pathology and imaging database between January 2006 and December 2016 with key words NAFLD, hepatic steatosis or steatohepatitis and HCC or hepatocellular carcinoma. This yielded a cohort of 400 patients. Among these 400 patients, only 38 patients met the AASLD criteria for $\mathrm{NAFLD}^{[15]}$ and had a triple phasic CT (late arterial, portal venous and delayed phase) before histological confirmation of HCC. Patients with cryptogenic cirrhosis were excluded due to uncertainty of the underlying etiology. Among the final group of 38 patients, 24 had NASH cirrhosis (cirrhosis with current or past evidence of steatosis or steatohepatitis) and 14 patients had no cirrhosis- 7 NASH (hepatic steatosis $\geq 5 \%$ with inflammation \pm fibrosis) and 7 NAFL (hepatic steatosis $\geq 5 \%$ without evidence of hepatocellular injury or fibrosis). A flowchart detailing the patient selection and subgroups is shown in Figure 1.

Patient age, sex, height, weight, body mass index (BMI), serum cholesterol, serum triglycerides, presence or absence of obesity, tumor histopathological and clinical management information were obtained from electronic medical records.

Cytological and pathological TNM staging was evaluated according to criteria of the 7th American Joint Committee on Cancer ${ }^{[26]}$. The diagnosis of NAFL, NASH was established at pathology. The time interval between CT and surgical pathology was $137 \pm 387$ days (range 3 to 1802 days). Twenty-four cases had surgical pathology within 6 months of CT. Histology of the HCC and background liver was evaluated by an experienced pathologist (TM) with expertise in NAFLD. HCCs were graded based on WHO classification and NAFLD was graded based on NAS score.

\section{CT imaging review}

All the CT images were independently reviewed on PACS Workstation (Centricity, GE Healthcare, Waukesha, WI) by four board-certified abdominal radiologists (SPS, ECE, AK, CAB) who were blinded to clinical and pathological findings other than the presence of HCC. Each reader recorded the imaging features of HCC, including size, location, APHE, PVWO, DPWO, and presence capsule. Readers also assessed for findings of cirrhosis-surface nodularity, caudate lobe hypertrophy, left lobe enlargement, widened fissures, widened gallbladder fossa, and portal hypertension ( $\mathrm{PH})$, splenomegaly, collaterals 
and gastroesophageal varices. In patients with multiple HCCs, only the largest HCC with histological confirmation was assessed. The final imaging features of HCCs were determined by majority. A fifth reader blinded to clinical and pathological findings reviewed cases lacking majority. LIRADs criteria is only applicable in patients with cirrhosis or chronic viral hepatitis and therefore would not be applicable for patients with NAFLD without cirrhosis.

\section{Statistical analysis}

Continuous variables were expressed as mean \pm standard deviation and categorical data as percentage. Inter-rater agreement was determined by prevalence-adjusted bias-adjusted Cohen's kappa ${ }^{[27]}$. Agreement between cirrhotic on CT and pathology was also determined by prevalence-adjusted Cohen's kappa ${ }^{[27]}$. Inter observer agreement was classified as none (0-0.2), minimal (0.21-0.39), weak (0.40-0.59), moderate (0.60-0.79) and strong (0.80-0.90) and almost perfect (>0.90). Differences between non-cirrhotic and cirrhotic subgroups were compared using an unpaired $t$-test with equal variance assumption for continuous data and Fischer's exact test for categorical data. Statistical significance was assumed for $P$ values of less than 0.05 . Data were analyzed using JMP 11.0 (SAS, Cary, NC) and Prism 5.0 (GraphPad Software, Inc, La Jolla, CA).

\section{RESULTS}

\section{Clinical and pathological characteristics}

Mean age of subjects was 63 years (range 45-79). Patients were predominantly male $(n=26,68.4 \%)$, diabetic $(n=28,73.7 \%)$, and obese (BMI $\geq 30 ; n=25,65.8 \%)$. Mean \pm SD total serum cholesterol and triglyceride values were $151.9 \pm 39.7$ (range: $36-229 \mathrm{mg} / \mathrm{dL}$ ) and $130.6 \pm 53.5$ (range: $54-237 \mathrm{mg} / \mathrm{dL}$ ), respectively. Most of the patients underwent liver transplant $(n=21,55.3 \%)$ or hepatic resection or segmentectomy $(n=12$, $31.6 \%$ ). Of the 21 patients that underwent liver transplant, 16 patients received chemoembolization (14) or ablation (2) before transplantation, 4 patients received only ablation or chemoembolization, and 1 patient was lost to follow-up. Of 38 patients, 2 (5.3\%) patients developed recurrent HCC. CT studies performed before any treatment was used for imaging analysis.

At pathology, HCCs were well differentiated in 14 patients (36.8\%), well to moderately differentiated in 5 patients $(13.2 \%)$, moderately differentiated in 16 patients $(42.1 \%)$, moderate to poorly differentiated in 2 patients $(5.3 \%)$ and poorly differentiated in 1 patient (2.6\%). Hepatic steatosis was minimal (26.3\%) in 10 patients $(26.3 \%)$, mild in 26 patients (68.4\%), and moderate in 2 patients (5.3\%). Steatohepatitis was present in 25 patients $(65.8 \%)$ and cirrhosis in 24 patients $(63.2 \%)$. The clinical and pathological characteristics are summarized in Table 1.

\section{$\mathrm{HCC}$ and liver parenchyma imaging characteristics - majority features}

Mean HCC size was $3.6 \pm 2.8 \mathrm{~cm}$ (range: $1.1-16.0 \mathrm{~cm}$ ). APHE was seen in $92.1 \%$, PVWO in $55.3 \%$ DPWO in $81.6 \%$ and enhancing capsule in $44.7 \%$ [Figures 2 and 3]. Fat within the HCC was present in only one patient. Cirrhotic morphology was present in 25 patients (65.8\%) and portal hypertension in 24 patients (63.2\%). The imaging features are summarized in Table 2.

\section{Non-cirrhotic NAFLD vs. cirrhotic NAFLD}

Non-Cirrhotic NAFLD (NAFL in 7 and NASH in 7) and cirrhotic NAFLD were present in 14 (36.8\%) and $24(63.2 \%)$ patients respectively. Patients with non-cirrhotic NAFLD were older $(P=0.03)$, had larger mean HCC size $(P=0.008)$ and higher degree of hepatic steatosis $(P=0.003)$. PVWO feature was observed significantly more in the non-cirrhotic group as compared to the cirrhotic group (78.6\% vs. $41.7 \%, P=0.04)$. Portal hypertension features were more commonly seen in patients with cirrhotic NAFLD $(91.7 \%$ vs. $14.3 \%$, $P<0.0001)$. There was no significant difference between the two groups with respect to gender distribution $(P=0.47)$, BMI $(P=0.14)$, presence of diabetes $(P=0.45)$, cholesterol level $(P=0.24)$, triglyceride level 
Table 1. Clinical-pathological characteristics in patients with NAFLD-associated HCC $(n=38 ;$ main $)$ by non-cirrhotic $(n=14)$ and cirrhotic $(n=24)$ liver

\begin{tabular}{|c|c|c|c|c|}
\hline Clinical and Pathological findings & Main Cohort $(n=38)$ & Non-cirrhotic $(n=14)$ & Cirrhotic $(n=24)$ & $P$-value \\
\hline Age (years) (mean \pm SD) & $63 \pm 7.2$ & $66.2 \pm 6.3$ & $60.9 \pm 7.2$ & 0.03 \\
\hline Gender & & & & 0.47 \\
\hline Female & $12(31.6)$ & $3(21.4)$ & $9(37.5)$ & \\
\hline Male & $26(68.4)$ & $11(78.6)$ & $15(62.5)$ & \\
\hline $\mathrm{BMI}\left(\mathrm{kg} / \mathrm{m}^{2}\right)($ mean $\pm \mathrm{SD})$ & $32.2 \pm 4.9$ & $30.6 \pm 4.6$ & $33.1 \pm 5.0$ & 0.14 \\
\hline Diabetes (\%) & $28(73.7)$ & $9(64.3)$ & $19(79.2)$ & 0.45 \\
\hline Total Cholesterol (mean \pm SD) & $151.9 \pm 39.7$ & $171 \pm 36.4$ & $147.4 \pm 39.9$ & 0.24 \\
\hline Triglycerides (mean \pm SD) & $130.6 \pm 53.5$ & $129.2 \pm 71.1$ & $131 \pm 50.7$ & 0.95 \\
\hline \multicolumn{5}{|l|}{ HCC Pathology Source } \\
\hline Biopsy & $5(13.2)$ & $3(21.4)$ & $2(8.3)$ & $<0.001$ \\
\hline Surgical Resection & $12(31.6)$ & $10(71.4)$ & $2(8.3)$ & \\
\hline Liver Explant at Transplant & $21(55.3)$ & $1(7.1)$ & $20(83.3)$ & \\
\hline \multicolumn{5}{|l|}{ Pathologic Features } \\
\hline \multicolumn{5}{|l|}{ Tumor Grade } \\
\hline Well differentiated & $14(36.8)$ & $4(29)$ & $10(41.7)$ & 0.31 \\
\hline Well-Moderately differentiated & $5(13.2)$ & $1(7.1)$ & $4(16.7)$ & \\
\hline Moderately differentiated & $16(42.1)$ & $9(64.3)$ & $7(29.2)$ & \\
\hline Moderate-Poorly differentiated & $2(5.3)$ & & $2(8.3)$ & \\
\hline Poorly differentiated & $1(2.6)$ & & $1(4.2)$ & \\
\hline Hepatic Steatosis Grade & & & & 0.003 \\
\hline Minimal & $10(26.3)$ & & $10(41.7)$ & \\
\hline Mild & $26(68.4)$ & $12(85.7)$ & $14(58.3)$ & \\
\hline Moderate & $2(5.3)$ & $2(14.3)$ & & \\
\hline \multicolumn{5}{|l|}{ Hepatic Fibrosis Stage } \\
\hline 0 & $9(23.7)$ & $9(64.3)$ & & \\
\hline 1 & $3(7.9)$ & $3(21.4)$ & & \\
\hline 2 & $2(5.3))$ & $2(14.3)$ & & \\
\hline 4 & $24(63.2)$ & & $24(100)$ & \\
\hline
\end{tabular}

$P$-value for non-cirrhotic group $v s$. cirrhotic group; continuous variables analyzed using unpaired $t$-test with equal variance assumption; categorical data analyzed using Fischer's exact test. BMI: body mass index; NAFLD: non-alcoholic fatty liver disease; HCC: hepatocellular carcinoma

$(P=0.95)$, tumor size $(P=0.67)$, tumor grade $(P=0.31)$, APHE $(P=1.00)$, DPWO $(P=1.00)$ and enhancing capsule $(P=0.09)$.

\section{HCC and liver parenchyma imaging characteristics - inter-rater agreement}

Inter-rater agreement was moderate to almost perfect for HCC APHE (0.74-1.0), none to moderate for PVWO (0-0.42), weak to almost perfect for DPWO (0.47-0.95) [Figure 4] and none to moderate for capsule (0.05-0.79). The inter-rater agreement was moderate to almost perfect for cirrhosis (0.79-0.89) and portal hypertension (0.79-0.95). Pathology and CT agreement for presence of cirrhosis was strong at 0.84. Interrater agreement for imaging features is summarized in Table 3.

\section{DISCUSSION}

In our study of NAFLD associated HCC, many HCCs did not demonstrate major imaging features particularly PVWO and enhancing capsule were absent in nearly half and DPWO was absent in nearly $20 \%$ of the patients. Cirrhotic morphology was present in 25 (65.8\%) patients. A third (36.8\%) of HCC occurring in non-cirrhotic livers would not be eligible for LIRADs classification.

Our study results are in agreement with previous reports in literature that HCC can occur even in the absence of steatohepatitis or fibrosis/cirrhosis ${ }^{[10,19-21,28-34]}$. Also HCCs in non-cirrhotic liver were larger in agreement with existing literature that HCCs in non-cirrhotic liver usually present at a later stage and are 

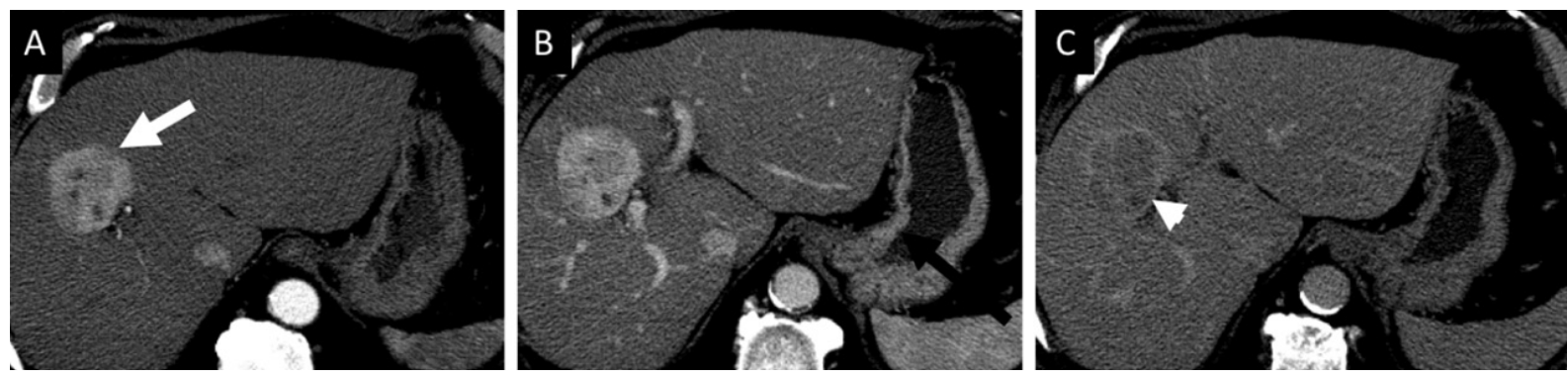

Figure 2. A 70-year old male with histologically confirmed hepatocellular carcinoma in non-cirrhotic liver with mild steatosis and no steatohepatitis (non-alcoholic fatty liver). Patient was obese (BMI of 30.7), diabetic and dyslipidemic. On multiphase contrast enhanced CT (A-C), a heterogeneously enhancing mass (arrow) on arterial phase (A) with no washout on portal venous (B) and complete washout on delayed phase (C) images can be seen. In addition, a thin but incomplete capsule (arrowhead) can be seen on delayed phase image (C). No features of portal hypertension were seen on imaging
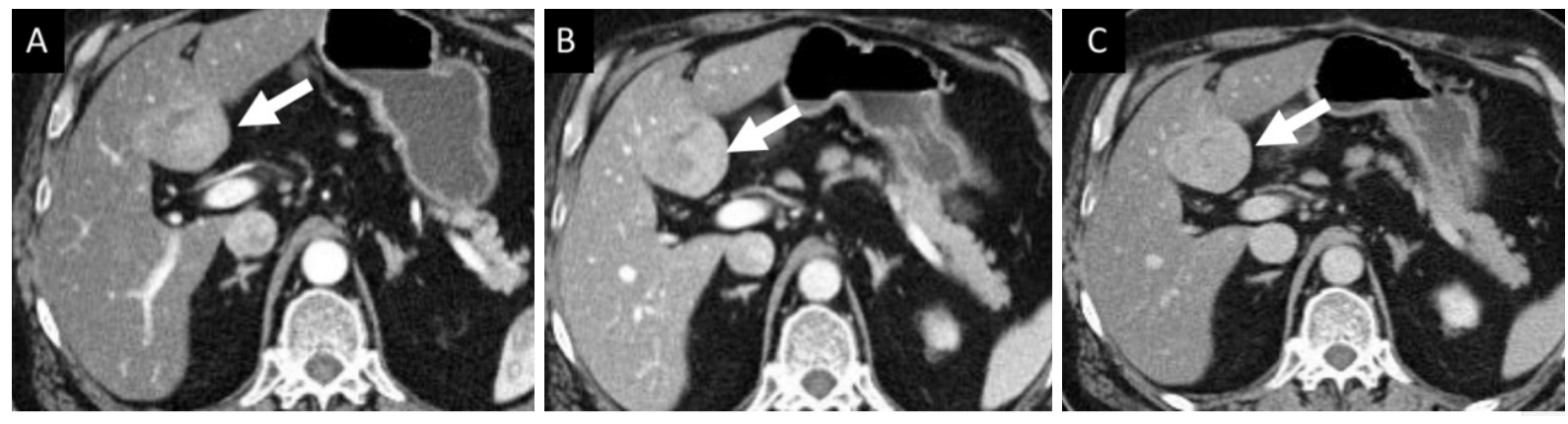

Figure 3. A 73-year old male with histologically confirmed hepatocellular carcinoma in non-cirrhotic liver with severe steatohepatitis (non-alcoholic steatohepatitis). Patient was obese (BMI of 31.1) and diabetic. On multiphase contrast enhanced CT (A-C), a heterogeneously enhancing mass (arrow) on arterial phase (A) showing no washout on portal venous (B) and delayed phase (C) images can be seen

Table 2. Imaging features of HCC and liver parenchyma at CT by majority consensus in patients with NAFLD-associated HCC $(n=38)$ by non-cirrhotic $(n=14)$ and cirrhotic $(n=24)$ liver morphology

\begin{tabular}{lcccc}
\hline HCC imaging features & Main cohort $(\boldsymbol{n}=\mathbf{4 8})$ & Non-cirrhotic pathology $(\boldsymbol{n}=\mathbf{1 4})$ & Cirrhotic pathology $(\boldsymbol{n}=\mathbf{2 4})$ & $\boldsymbol{P}$-value \\
\hline Tumor size (mean \pm SD) & $3.6 \pm 2.8$ & $5.1 \pm 3.9$ & $2.7 \pm 1.3$ & 0.008 \\
APHE & $35(92.1)$ & $13(92.8)$ & $22(91.7)$ & 1.00 \\
PVWO & $21(55.3)$ & $11(78.6)$ & $10(41.7)$ & 0.04 \\
DPWO & $31(81.6)$ & $12(85.7)$ & $19(79.2)$ & 1.00 \\
Enhancing "Capsule" & $17(44.7)$ & $9(64.3)$ & $8(33.3)$ & 0.09 \\
Cirrhotic Liver Morphology & $25(65.8)$ & $2(14.3)$ & $23(95.8)$ & $<0.001$ \\
Portal hypertension & $24(63.2)$ & $2(14.3)$ & $22(91.7)$ & $<0.001$ \\
LIRADS Score* & & & & 0.29 \\
2 & $3(7.9)$ & 0 & 0 & \\
3 & 0 & 0 & $8(33.3)$ & $13(54.2)$ \\
4 & $11(28.9)$ & $3(21.4)$ & $11(78.6)$ & \\
5 & $24(63.2)$ & &
\end{tabular}

* LIRADS score applied to see if HCCs would meet the criteria, however if there is no cirrhosis, LIRADs criteria should not be applied as per guidelines. APHE: arterial phase hyperenhancement; PVWO: portal venous washout; DPWO: delayed phase washout

larger in size at presentation ${ }^{[20]}$. This may be due to lack of screening guidelines in non-cirrhotic patients with NAFLD ${ }^{[3,28]}$. Larger tumor size seen in noan-cirrhotic livers at presentation may make them ineligible for transplant based on Milan criteria for transplantation ${ }^{[35,36]}$.

Imaging features of HCC on multiphase CT and MRI are based on sequential changes in the intra-lesional blood supply during hepatocarcinogenesis. Advanced HCCs receive their blood supply predominantly 

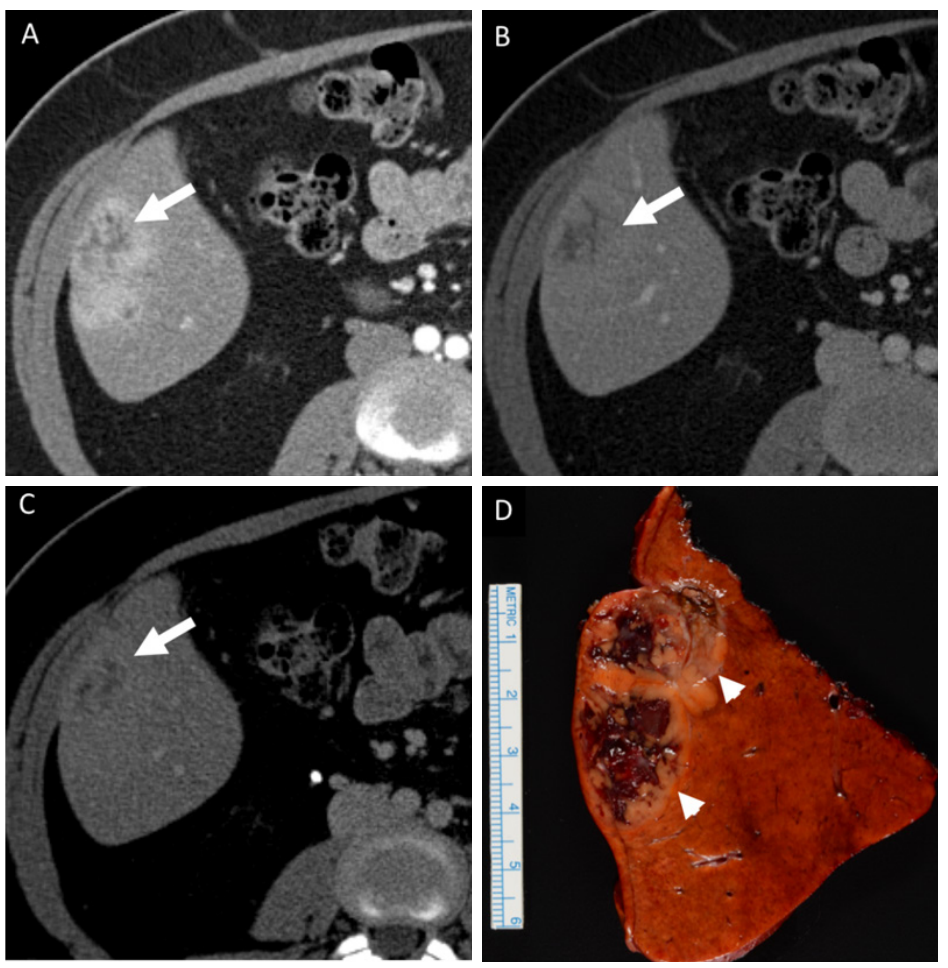

Figure 4. A 47-year old female with histologically confirmed hepatocellular carcinoma (HCC) in non-cirrhotic liver with minimal steatosis and moderate steatohepatitis (non-alcoholic steatohepatitis). Patient was obese (BMI of 30.5) and diabetic. On multiphase contrast enhanced CT (A-C), three of the four readers interpreted this as a heterogeneously enhancing mass (arrow) on arterial phase (A) with no washout on portal venous (B) and delayed phase (C) images. However lof the four readers interpreted this as a heterogeneously enhancing mass (arrow) on arterial phase (A) with washout on portal venous (B) and delayed phase (C) images. A resected specimen (D) showing HCC (arrow) with background fatty liver can be seen

Table 3. Inter-observer agreement for HCC features and liver parenchyma morphology at MRI in patients with NAFLDassociated HCC $(n=38)$

\begin{tabular}{lcccccc}
\hline HCC imaging features & R1 vs. R2 & R1 vs. R3 & R1 vs. R4 & R2 vs. R3 & R2 vs. R4 & R3 vs. R4 \\
\hline APHE & 0.74 & 0.79 & 0.79 & 0.74 & 0.84 & 1.0 \\
PVWO & 0.42 & 0.00 & 0.53 & 0.05 & 0.32 & 0.05 \\
DPWO & 0.95 & 0.84 & 0.74 & 0.79 & 0.68 & 0.47 \\
Enhancing "Capsule" & 0.47 & 0.05 & 0.42 & 0.37 & 0.79 & 0.37 \\
Cirrhotic Liver Morphology & 0.79 & 0.89 & 0.89 & 0.79 & 0.79 & 0.89 \\
Portal hypertension & 0.74 & 0.79 & 0.95 & 0.84 & 0.79 & 0.84 \\
\hline
\end{tabular}

Data are presented as prevalence-adjusted bias-adjusted kappa. R1: reader 1; R2: reader 2; R3: reader 3; R4: reader 4; APHE: arterial phase hyperenhancement; PVWO: portal venous phase washout; DPWO: delayed phase washout

from the anomalous arteries (arterial blood supply) ${ }^{[37,38]}$. This results in high arterial flow which manifests as APHE on dynamic multiphase imaging followed by washout (de-enhancement of a HCC, greater enhancement of the surrounding liver, or a combination of both factors) on portal venous and/or delayed phase imaging ${ }^{[39-43]}$. The washout is attributed to diminished portal venous blood supply of the HCC, high tumoral cellularity with associated small extracellular volume, and expanded extracellular space of the surrounding fibrotic liver ${ }^{[37,38,43,44]}$.

Effect of NAFLD on imaging features on HCC is currently being explored. In our study APHE was present in most (92.1\%) of the cases. APHE is a major imaging criterion and has a good sensitivity for detection of HCC, ranging from $65 \%-9 \%^{[39,43,45]}$. The lower sensitivity can be seen in early/well differentiated HCC with partial neovascularization. APHE is more sensitive than other enhancement features but lacks specificity for 
HCC. The specificity can be increased by combined assessment of APHE \& washout (PVWO or DPWO) ${ }^{[39,46]}$. Sensitivity and specificity of combined APHE \& washout for diagnosis of HCC ranges from 43\% to 98\%, and $81 \%$ to $100 \%$, respectively ${ }^{[43,46-48]}$.

For assessment of washout, either portal venous or delayed phase imaging can be used. In this study, PVWO was absent in 17 (44.7\%) of the HCCs. In contrast, DPWO was absent in only 7 (18.4\%) HCCs. In addition, the interobserver agreement was none to moderate for PVWO and weak to almost perfect for DPWO. These results support the added value of delayed phase imaging in assessment of washout. Triple phase CT is the standard protocol for HCC in chronic liver disease, however some centers may not include the delayed phase to reduce the radiation dose ${ }^{[4]}$. However, this may lead to loss of important diagnostic information (washout characteristics). In a prior study with NAFLD associated HCC, PVWO was absent in $30 \%$ cases but DPWO was not reported ${ }^{[49]}$. Ehman et al. ${ }^{[45]}$ reported lack of washout (portal or delayed) in only $18 \%$ ( $15 \%$ on CT and $21 \%$ on MRI) of the 184 pathologically proven HCCs with cirrhosis resulting from several different etiologies ${ }^{[45]}$. Washout in NAFLD associated HCC may not be demonstrated well probably due to hepatic steatosis that can lead to liver hypo-attenuation on post-contrast enhanced images, thereby resulting in the appearance of persistent HCC hyper-attenuation or iso-attenuation during the portal venous and delayed phase imaging. Interestingly however in our study PVWO was more commonly seen in subgroup with non-cirrhotic NAFLD, which had higher proportion of cases with mild moderate steatosis in comparison to cirrhotic NAFLD subgroup. These findings suggest that degree of hepatic steatosis may have more determining effect than presence of fibrosis for washout appearance. Future studies with larger number of patients are required to confirm our findings. The findings should be confirmed in studies with larger population of NAFLD associated HCCs.

Capsule appearance is a highly specific but not very sensitive feature of $\operatorname{HCC}^{[43,45,50]}$. Capsule is thought to be caused by expansile growth of HCC causing perilesional compression of liver tissue, which appears as enhancing rim around the HCC in portal venous or delayed phase. Capsule is supplied by the portal venous system leading to this delayed enhancement ${ }^{[51]}$. Interestingly, capsule is a recognized major feature of HCC based on the LIRADS, OPTN guidelines but not on the AASLD and EASL guidelines ${ }^{[7,8]}$. In our study, capsule appearance was seen in only 17 (44.7\%) of the NAFLD-associated-HCCs but still within the reported range of 27 to $64 \%$ in studies including all chronic liver diseases ${ }^{[43,45]}$.

Inter-rater agreement was poor for PVWO, variable for DPWO and capsule suggesting the difficulty in interpretation of washout and detection of capsule in NAFLD associated HCCs [Figure 4]. We had four experienced abdominal radiologists working in tertiary level institute with large exposure to HCCs on a daily basis. Even with this level of expertise, the interobserver agreement for PVWO was poor. We think that this is due to the variable hepatic steatosis in background liver that affects interpretation of the washout particularly PVWO.

The study has some limitations. Despite searching a large patient database containing around 2,500 patients (pathological data for HCC), our final study cohort was relatively small. Due to retrospective nature of the study, the imaging (triphasic CT) technique including scanner, sequence protocol(s) and contrast agent(s) was not uniform over the study period and this may have introduced variability in the phase of image acquisition. Some cases of NAFLD associated HCCs may have been excluded that either lacked pathological assessment or were interpreted as cryptogenic cirrhosis. The readers were aware of the diagnosis of HCC in the lesions which may have introduced bias for imaging features. However this bias of prior knowledge did not inflate interobserver agreement for the imaging features. Degree of hepatic steatosis may have changed from time between histological analysis and radiological assessment, due to systemic interventions or natural progression of NAFLD. This was unavoidable as several patients received locoregional treatment before undergoing surgery or liver transplantation. These treatments may also have 
contributed to the changes in the liver parenchyma. However we minimized this variation as most of the studies had histological evaluation within 6 months of CT study.

In conclusion, NAFLD associated HCC may not show portal venous phase washout on CT and may impact the imaging diagnosis of HCC. Our study should be confirmed in studies with larger population of NAFLD associated HCCs. There may be a need for modification of criteria for multiphase CT based diagnosis of NAFLD associated HCCs particularly in the non-cirrhotic patients.

\section{DECLARATIONS}

\section{Authors' contributions}

Made substantial contributions to conception and design of the study and performed data analysis and interpretation: Garg I, Thompson SM, Mounajjed T, Ehman EC, Venkatesh SK

Performed data acquisition, as well as provided administrative, technical, and material support: Garg I, Thompson SM, Sheedy SP, Mounajjed T, Khandelwal A, Ehman EC, Bookwalter CA, Venkatesh SK

\section{Availability of data and materials}

Not applicable.

\section{Financial support and sponsorship}

None.

\section{Conflicts of interest}

All authors declared that there are no conflicts of interest.

\section{Ethical approval and consent to participate}

In this IRB approved (ID: 15-004925), HIPPA-compliant retrospective study. Written informed consent waived by the IRB.

\section{Consent for publication}

Not applicable.

\section{Copyright}

(c) The Author(s) 2019.

\section{REFERENCES}

1. Global Burden of Disease Liver Cancer Collaboration, Akinyemiju T, Abera S, Ahmed M, Alam N, et al. The burden of primary liver cancer and underlying etiologies from 1990 to 2015 at the global, regional, and national level: results from the global burden of disease study 2015. JAMA Oncol 2017;3:1683-91.

2. Torre LA, Bray F, Siegel RL, Ferlay J, Lortet-Tieulent J, et al. Global cancer statistics, 2012. CA Cancer J Clin 2015;65:87-108.

3. Bruix J, Sherman M, American Association for the Study of Liver D. Management of hepatocellular carcinoma: an update. Hepatology 2011;53:1020-2.

4. Choi JY, Lee JM, Sirlin CB. CT and MR imaging diagnosis and staging of hepatocellular carcinoma: part I. Development, growth, and spread: key pathologic and imaging aspects. Radiology 2014;272:635-54.

5. European Association For The Study Of The Liver; European Organisation For Research And Treatment Of Cancer. EASL-EORTC clinical practice guidelines: management of hepatocellular carcinoma. J Hepatol 2012;56:908-43.

6. Leoni S, Piscaglia F, Golfieri R, Camaggi V, Vidili G, et al. The impact of vascular and nonvascular findings on the noninvasive diagnosis of small hepatocellular carcinoma based on the EASL and AASLD criteria. Am J Gastroenterol 2010;105:599-609.

7. Heimbach JK, Kulik LM, Finn RS, Sirlin CB, Abecassis MM, et al. AASLD guidelines for the treatment of hepatocellular carcinoma. Hepatology 2018;67:358-80.

8. Wald C, Russo MW, Heimbach JK, Hussain HK, Pomfret EA, et al. New OPTN/UNOS policy for liver transplant allocation: standardization of liver imaging, diagnosis, classification, and reporting of hepatocellular carcinoma. Radiology 2013;266:376-82. 
9. El-Serag HB, Rudolph KL. Hepatocellular carcinoma: epidemiology and molecular carcinogenesis. Gastroenterology 2007;132:2557-76.

10. Ertle J, Dechene A, Sowa JP, Penndorf V, Herzer K, et al. Non-alcoholic fatty liver disease progresses to hepatocellular carcinoma in the absence of apparent cirrhosis. Int J Cancer 2011;128:2436-43.

11. Malik SM, Gupte PA, de Vera ME, Ahmad J. Liver transplantation in patients with nonalcoholic steatohepatitis-related hepatocellular carcinoma. Clin Gastroenterol Hepatol 2009;7:800-6.

12. Yang JD, Kim B, Sanderson SO, St Sauver JL, Yawn BP, et al. Hepatocellular carcinoma in olmsted county, Minnesota, 1976-2008. Mayo Clin Proc 2012;87:9-16.

13. Browning JD, Szczepaniak LS, Dobbins R, Nuremberg P, Horton JD, et al. Prevalence of hepatic steatosis in an urban population in the United States: impact of ethnicity. Hepatology 2004;40:1387-95.

14. Perumpail BJ, Khan MA, Yoo ER, Cholankeril G, Kim D, et al. Clinical epidemiology and disease burden of nonalcoholic fatty liver disease. World J Gastroenterol 2017;23:8263-76.

15. Chalasani N, Younossi Z, Lavine JE, Charlton M, Cusi K, et al. The diagnosis and management of nonalcoholic fatty liver disease: practice guidance from the American association for the study of liver diseases. Hepatology 2018;67:328-57.

16. Caldwell SH, Oelsner DH, Iezzoni JC, Hespenheide EE, Battle EH, et al. Cryptogenic cirrhosis: clinical characterization and risk factors for underlying disease. Hepatology 1999;29:664-9.

17. Poonawala A, Nair SP, Thuluvath PJ. Prevalence of obesity and diabetes in patients with cryptogenic cirrhosis: a case-control study. Hepatology 2000;32:689-92.

18. Thuluvath PJ, Kantsevoy S, Thuluvath AJ, Savva Y. Is cryptogenic cirrhosis different from NASH cirrhosis? J Hepatol 2018;68:519-25.

19. Guzman G, Brunt EM, Petrovic LM, Chejfec G, Layden TJ, et al. Does nonalcoholic fatty liver disease predispose patients to hepatocellular carcinoma in the absence of cirrhosis? Arch Pathol Lab Med 2008;132:1761-6.

20. Leung C, Yeoh SW, Patrick D, Ket S, Marion K, et al. Characteristics of hepatocellular carcinoma in cirrhotic and non-cirrhotic nonalcoholic fatty liver disease. World J Gastroenterol 2015;21:1189-96.

21. Paradis V, Zalinski S, Chelbi E, Guedj N, Degos F, et al. Hepatocellular carcinomas in patients with metabolic syndrome often develop without significant liver fibrosis: a pathological analysis. Hepatology 2009;49:851-9.

22. American College of Radiology. Liver Imaging and Reporting and Data System. CT/MRI LI-RADS v2017. Available from https:// www.acr.org/Clinical-Resources/Reporting-and-Data-Systems/LI-RADS/CT-MRI-LI-RADS-v2017. [Last accessed on Oct 15 2019]

23. Hamer OW, Aguirre DA, Casola G, Lavine JE, Woenckhaus M, et al. Fatty liver: imaging patterns and pitfalls. Radiographics 2006;26:1637-53.

24. Kodama Y, Ng CS, Wu TT, Ayers GD, Curley SA, et al. Comparison of CT methods for determining the fat content of the liver. AJR Am J Roentgenol 2007;188:1307-12.

25. Ma X, Holalkere NS, Kambadakone RA, Mino-Kenudson M, Hahn PF, et al. Imaging-based quantification of hepatic fat: methods and clinical applications. Radiographics 2009;29:1253-77.

26. Edge SB; American Joint Committee on Cancer. AJCC cancer staging manual. New York: Springer; 2010.

27. Byrt T, Bishop J, Carlin JB. Bias, prevalence and kappa. J Clin Epidemiol 1993;46:423-9.

28. Baffy G, Brunt EM, Caldwell SH. Hepatocellular carcinoma in non-alcoholic fatty liver disease: an emerging menace. J Hepatol 2012;56:1384-91.

29. Bralet MP, Regimbeau JM, Pineau P, Dubois S, Loas G, et al. Hepatocellular carcinoma occurring in nonfibrotic liver: epidemiologic and histopathologic analysis of 80 French cases. Hepatology 2000;32:200-4.

30. Brancatelli G, Federle MP, Grazioli L, Carr BI. Hepatocellular carcinoma in noncirrhotic liver: CT, clinical, and pathologic findings in 39 U.S. residents. Radiology 2002;222:89-94.

31. Nzeako UC, Goodman ZD, Ishak KG. Hepatocellular carcinoma in cirrhotic and noncirrhotic livers. A clinico-histopathologic study of 804 North American patients. Am J Clin Pathol 1996;105:65-75.

32. Pinero F, Pages J, Marciano S, Fernandez N, Silva J, et al. Fatty liver disease, an emerging etiology of hepatocellular carcinoma in Argentina. World J Hepatol 2018;10:41-50.

33. Trevisani F, Frigerio M, Santi V, Grignaschi A, Bernardi M. Hepatocellular carcinoma in non-cirrhotic liver: a reappraisal. Dig Liver Dis 2010;42:341-7.

34. Yasui K, Hashimoto E, Komorizono Y, Koike K, Arii S, et al. Characteristics of patients with nonalcoholic steatohepatitis who develop hepatocellular carcinoma. Clin Gastroenterol Hepatol 2011;9:428-33; quiz e50.

35. Martin P, DiMartini A, Feng S, Brown R Jr, Fallon M. Evaluation for liver transplantation in adults: 2013 practice guideline by the American Association for the Study of Liver Diseases and the American Society of Transplantation. Hepatology 2014;59:1144-65.

36. Mazzaferro V, Regalia E, Doci R, Andreola S, Pulvirenti A, et al. Liver transplantation for the treatment of small hepatocellular carcinomas in patients with cirrhosis. N Engl J Med 1996;334:693-9.

37. Matsui O. Imaging of multistep human hepatocarcinogenesis by CT during intra-arterial contrast injection. Intervirology 2004;47:271-6.

38. Park YN, Yang CP, Fernandez GJ, Cubukcu O, Thung SN, et al. Neoangiogenesis and sinusoidal "capillarization" in dysplastic nodules of the liver. Am J Surg Pathol 1998;22:656-62.

39. Jang HJ, Kim TK, Khalili K, Yazdi L, Menezes R, et al. Characterization of 1-to 2-cm liver nodules detected on hcc surveillance ultrasound according to the criteria of the American Association for the Study of Liver Disease: is quadriphasic CT necessary? AJR Am J Roentgenol 2013;201:314-21.

40. Park MJ, Kim YK, Lee MH, Lee JH. Validation of diagnostic criteria using gadoxetic acid-enhanced and diffusion-weighted MR imaging for small hepatocellular carcinoma $(<=2.0 \mathrm{~cm})$ in patients with hepatitis-induced liver cirrhosis. Acta Radiol 2013;54:127-36. 
41. Ramalho M, Matos AP, AlObaidy M, Velloni F, Altun E, et al. Magnetic resonance imaging of the cirrhotic liver: diagnosis of hepatocellular carcinoma and evaluation of response to treatment - Part 1. Radiol Bras 2017;50:38-47.

42. Rimola J, Forner A, Tremosini S, Reig M, Vilana R, et al. Non-invasive diagnosis of hepatocellular carcinoma $</=2 \mathrm{~cm}$ in cirrhosis. Diagnostic accuracy assessing fat, capsule and signal intensity at dynamic MRI. J Hepatol 2012;56:1317-23.

43. Tang A, Bashir MR, Corwin MT, Cruite I, Dietrich CF, et al. Evidence supporting LI-RADS major features for CT- and MR imagingbased diagnosis of hepatocellular carcinoma: a systematic review. Radiology 2018;286:29-48.

44. Marrero JA, Fontana RJ, Su GL, Conjeevaram HS, Emick DM, et al. NAFLD may be a common underlying liver disease in patients with hepatocellular carcinoma in the United States. Hepatology 2002;36:1349-54.

45. Ehman EC, Behr SC, Umetsu SE, Fidelman N, Yeh BM, et al. Rate of observation and inter-observer agreement for LI-RADS major features at CT and MRI in 184 pathology proven hepatocellular carcinomas. Abdom Radiol (NY) 2016;41:963-9.

46. Oliver JH 3rd, Baron RL, Federle MP, Rockette HE Jr. Detecting hepatocellular carcinoma: value of unenhanced or arterial phase CT imaging or both used in conjunction with conventional portal venous phase contrast-enhanced CT imaging. AJR Am J Roentgenol 1996;167:71-7.

47. Luca A, Caruso S, Milazzo M, Mamone G, Marrone G, et al. Multidetector-row computed tomography (MDCT) for the diagnosis of hepatocellular carcinoma in cirrhotic candidates for liver transplantation: prevalence of radiological vascular patterns and histological correlation with liver explants. Eur Radiol 2010;20:898-907.

48. Serste T, Barrau V, Ozenne V, Vullierme MP, Bedossa P, et al. Accuracy and disagreement of computed tomography and magnetic resonance imaging for the diagnosis of small hepatocellular carcinoma and dysplastic nodules: role of biopsy. Hepatology 2012;55:800-6.

49. Iannaccone R, Piacentini F, Murakami T, Paradis V, Belghiti J, et al. Hepatocellular carcinoma in patients with nonalcoholic fatty liver disease: helical CT and MR imaging findings with clinical-pathologic comparison. Radiology 2007;243:422-30.

50. Khan AS, Hussain HK, Johnson TD, Weadock WJ, Pelletier SJ, et al. Value of delayed hypointensity and delayed enhancing rim in magnetic resonance imaging diagnosis of small hepatocellular carcinoma in the cirrhotic liver. J Magn Reson Imaging 2010;32:360-6.

51. Ueda K, Matsui O, Kawamori Y, Nakanuma Y, Kadoya M, et al. Hypervascular hepatocellular carcinoma: evaluation of hemodynamics with dynamic CT during hepatic arteriography. Radiology 1998;206:161-6. 